\title{
Recherche in neuer Dimension
}

\author{
Wer e.Med bucht, dem steht die ganze Welt der e.Bibliothek offen. \\ Diese umfasst nicht nur sämtliche Inhalte der deutschsprachigen Zeit- \\ schriften von Springer Medizin, sondern ermöglicht auch die Recherche \\ in SpringerLink, einer der weltweit größten Online-Bibliotheken.
}

$\mathrm{K}$ eine Frage, Bibliotheken wie die des Trinity College in Dublin haben auch heute noch einen enormen Charme. Doch wer nicht als Tourist unterwegs ist, sondern in seinem beruflichen Alltag nach fundierten Informationen sucht, weiß die Vorteile des Internets zu schätzen. Schnell, einfach und von jedem Ort lassen sich die vorhandenen Informationen screenen. Wie gut das Ergebnis der Recherche ist, hängt allerdings von der Datenbank, der Brauchbarkeit der Suche, der Sortierbarkeit der Ergebnisse und den Zugriffsmöglichkeiten auf die gesammelten Informationen ab. Eine große Trefferliste gewährleistet nämlich noch lange keine Ausspielung von Volltexten oder garantiert eine hohe Qualität der Inhalte.

\section{Mit wenigen Klicks in die ganze Welt der Medizin}

Eine neue Dimension der Recherche eröffnet die „e.Bibliothek“, die jedem Abonnenten von e.Med zur Verfügung steht. Hier stellt Springer Medizin Inhalte aus sämtlichen medizinischen Fachge-

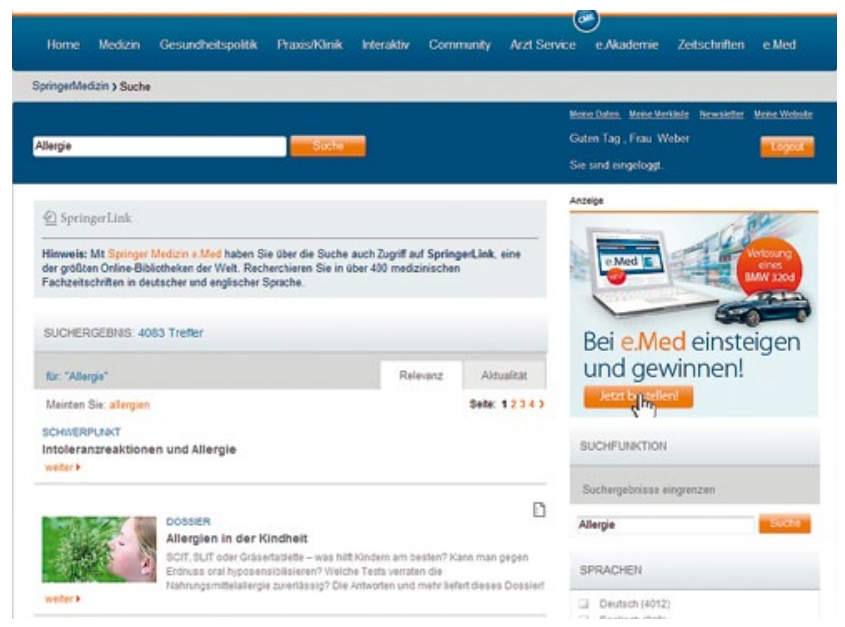

bieten zur Verfügung, also Publikationen aus allen hauseigenen Facharztmagazinen und wissenschaftlichen Zeitschriften. Alle Veröffentlichungen sind seriös recherchiert und geprüft. Das gilt für alle 470 Titel, die in der e.Bibliothek verfügbar sind - dafür stehen Herausgeber, Fachautoren und die Redaktionen von Springer Medizin gerade.

Mit e.Med haben Sie nicht nur Zugriff auf die Inhalte der deutschsprachigen Publikationen. Eine umfassende Suchfunktion auf dem Portal ermöglicht Ihnen auch die Recherche in den internationalen medizinischen Zeitschriften von SpringerLink. Sie finden somit die neuesten Veröffentlichungen aus aller Welt und können sich problemlos interdisziplinär informieren.

\section{Volltextsuche garantiert hohe Trefferquote}

Da die e.Bibliothek über eine Volltextsuche verfügt, liefern einzelne Suchbegriffe nicht zuletzt aufgrund der Größe des Angebots entsprechend viele Treffer. Doch keine Sorge, in der e.Bibliothek können

Beeindruckende 4.083 Treffer liefert z. B. der Suchbegriff "Allergie" in der e.Bibliothek. Verfeinern lässt sich die Suche durch Begriffskombinationen oder die Einschränkung auf Publikationen, Zeiträume oder Autoren etc.

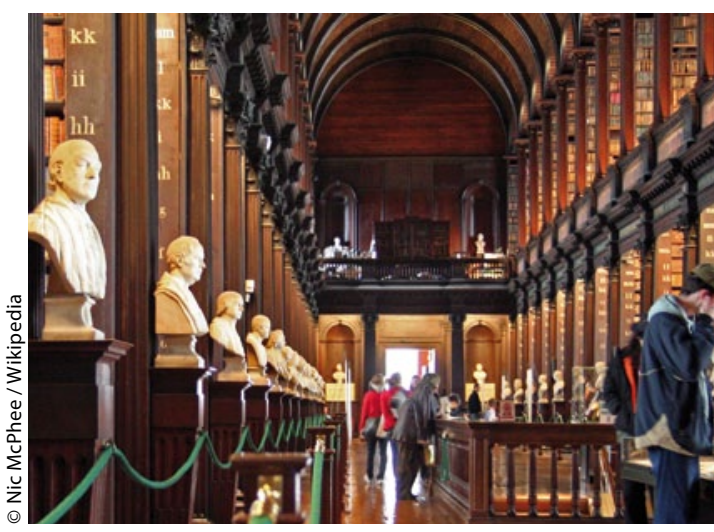

Von zeitlosem Charme: die 1732 gebaute Bibliothek des Trinity College

Sie Suchbegriffe kombinieren oder die Treffer nach Aktualität beziehungsweise Relevanz sortieren. Darüber hinaus lässt sich die Ergebnisliste auf bestimmte Zeitschriften, Zeiträume, Autoren etc. einschränken, sodass eine übersichtliche und individuelle Darstellung der Ergebnisse gewährleistet ist. Um Ihnen die Recherche zu erleichtern, gibt es einen weiteren Service: Sobald Sie einen Artikel aus der Trefferliste ausgewählt haben, öffnet sich das Fenster „Mehr zum Thema", in dem ähnliche beziehungsweise verwandte Beiträge angezeigt werden.

Und sollten Sie doch einmal eine PrintVersion benötigen, so ist auch das kein Problem: Alle Artikel sind als PDF download- und ausdruckbar.

\section{e.Med gratis testen}

Buchen und testen können Sie das e.MedPaket, das auch die e.Bibliothek beinhaltet, ganz einfach onlineunter der Adresse www.springermedizin.de/eMed. Dort klicken Sie auf ,e.Med bestellen“ oder „e.Med gratis testen" und folgen den Anweisungen.

Falls Sie noch Fragen zum e.Med-Paket haben, finden Sie unter www.springermedizin.de/faq die wichtigsten Antworten. Individuelle Unterstützung bietet darüber hinaus auch der Kundenservice telefonisch unter 08007780777 oder per E-Mail an kundenservice@ springermedizin.de 